\title{
Underwater Virtual Reality System for Neutral Buoyancy Training: Development and Evaluation
}

\author{
Christian B. Sinnott \\ csinnott@nevada.unr.edu \\ Department of Psychology, \\ University of Nevada, Reno \\ Savannah Halow \\ shalow@nevada.unr.edu \\ Department of Psychology, \\ University of Nevada, Reno \\ Jeffrey Mulligan \\ jeffrey.b.mulligan@nasa.gov \\ Human Systems Integration Division, \\ NASA Ames Research Center
}

\author{
James Liu \\ jliu@nevada.unr.edu \\ Department of Computer Science, \\ University of Nevada, Reno \\ Ann E. Jones \\ ajones@nevada.unr.edu \\ Department of Psychology, \\ University of Nevada, Reno \\ Michael A. Crognale
mcrognale@unr.edu
Department of Psychology,
University of Nevada, Reno \\ Paul R. MacNeilage \\ pmacneilage@unr.edu \\ Department of Psychology, \\ University of Nevada, Reno
}

\author{
Courtney Matera \\ cmatera@nevada.unr.edu \\ Department of Psychology, \\ University of Nevada, Reno \\ Matthew Moroz \\ mmoroz@nevada.unr.edu \\ Department of Psychology, \\ University of Nevada, Reno \\ Eelke Folmer \\ efolmer@unr.edu \\ Department of Computer Science, \\ University of Nevada, Reno
}

\begin{abstract}
During terrestrial activities, sensation of pressure on the skin and tension in muscles and joints provides information about how the body is oriented relative to gravity and how the body is moving relative to the surrounding environment. In contrast, in aquatic environments when suspended in a state of neutral buoyancy, the weight of the body and limbs is offloaded, rendering these cues uninformative. It is not yet known how this altered sensory environment impacts virtual reality experiences. To investigate this question, we converted a full-face SCUBA mask into an underwater head-mounted display and developed software to simulate jetpack locomotion outside the International Space Station. Our goal was to emulate conditions experienced by astronauts during training at NASA's Neutral Buoyancy Lab. A user study was conducted to evaluate both sickness and presence when using virtual reality in this altered sensory environment. We observed an increase in nausea related symptoms underwater, but we cannot conclude that this is due to VR use. Other measures of sickness and presence underwater were comparable to measures taken above water. We conclude with suggestions for improved underwater VR systems and improved methods for evaluation of these systems based on our experience.
\end{abstract}

\section{CCS CONCEPTS}

- Human-centered computing $\rightarrow$ Virtual reality; • Software and its engineering $\rightarrow$ Virtual worlds training simulations.

\section{KEYWORDS}

Virtual Reality, Simulation, Training, Space, Spacewalk, Underwater, Waterproof, Head-mounted Display, Sickness, Presence
ACM Reference Format:

Christian B. Sinnott, James Liu, Courtney Matera, Savannah Halow, Ann E. Jones, Matthew Moroz, Jeffrey Mulligan, Michael A. Crognale, Eelke Folmer, and Paul R. MacNeilage. 2019. Underwater Virtual Reality System for Neutral Buoyancy Training: Development and Evaluation. In 25th ACM Symposium on Virtual Reality Software and Technology (VRST '19), November 12-15, 2019, Parramatta, NSW, Australia. ACM, New York, NY, USA, 9 pages. https://doi.org/10.1145/3359996.3364272

\section{INTRODUCTION}

Astronauts are occasionally required to exit the spacecraft to perform maintenance or other extra-vehicular activities (EVA). Due to the risk and unfamiliarity of this situation and environment, astronauts train for EVA before they enter space. NASA's EVA training takes place in a large facility called the Neutral Buoyancy Lab (NBL). Here, astronauts put on pressurized spacesuits that have been modified for use underwater rather than in space. They are then submerged in a large pool along with replicas of spacecraft to create a physical and visual training environment that resembles the space environment as closely as possible. Astronauts train seven hours underwater for every one hour of anticipated EVA [National Aeronautics and Space Administration 2018].

There are many advantages of training at the NBL. For example, astronauts become accustomed to being confined in the bulky spacesuits. Perhaps more importantly, they experience the sensation of floating as they would in zero-gravity. This is achieved by maintaining neutral buoyancy, meaning that the astronauts do not float to the surface or sink to the bottom. Neutral buoyancy is a good analog for zero-gravity because common sensory cues to body orientation are rendered uninformative. These include somatosensory cues that provide information about pressure on the skin as well as proprioceptive cues that provide information about joint 


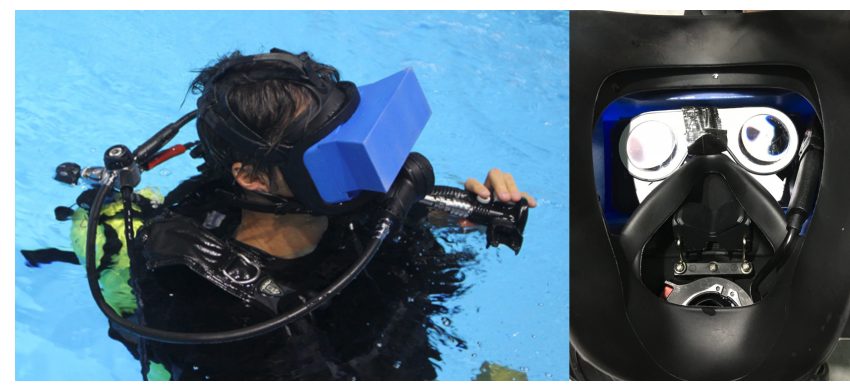

Figure 1: An underwater head mounted display (U-HMD) was created by adapting a full-face SCUBA mask to house a smartphone and lenses using a custom 3D printed insert.

articulation and muscle tension. Both types of cues normally provide information about how the weight of the body is supported. While underwater, only vestibular cues from the inner ear remain to provide reliable non-visual information about the direction of gravity. In this altered sensory environment, astronauts gain valuable experience not only maneuvering in the spacesuits, but also practicing novel locomotion methods.

While this training method has many advantages, there are several disadvantages as well, with the primary disadvantage being a large maintenance cost. The pool used at the NBL is one of the world's largest pools, with a capacity of 6.2 million gallons [National Aeronautics and Space Administration 2018]. Because this pool is kept at at constant temperature of $85 \mathrm{~F}(29.4 \mathrm{C})$, heating costs for this facility are significant. Full-time staff are required not only for maintenance, but also to ensure safety, with up to four divers required to accompany each astronaut during training [Lauchert and Narramore 2017]. In order to help cover costs, NASA regularly rents the facility out to other space agencies and private companies [Berger 2017]. Another large disadvantage is the lack of accessibility since few companies or agencies can afford access to this unique facility.

To overcome these disadvantages, we developed a low-cost underwater head-mounted display (U-HMD) (see Figure 1) that can be used in any normal pool. Users wearing Self-Contained Underwater Breathing Apparatus (SCUBA) equipment can be exposed to an altered sensory environment and experience the sensation of floating, similar to the NBL, but at a much reduced cost. The virtual reality headset allows for simulating any visual environment, eliminating the need for expensive physical replicas of spacecraft. In addition, with virtual reality, we can eliminate background visual cues to gravity that would be visible in the NBL, such as the bottom of the pool or the surface of the water. These can then be replaced with background scenes that are more consistent with the target training environment of space, which should enhance training presence.

To demonstrate proof of concept, we developed an application to train users to perform jetpack locomotion during neutral buoyancy. We chose this training task because locomotion is an important consideration for VR applications [Zayer, MacNeilage, and Folmer 2018], and locomotor tasks are ideal for assessing presence and sickness in VR. Our application mimics the interface and control dynamics of NASA's SAFER jetpack system; SAFER stands for Simplified Aid for EVA Rescue. SAFER is a self-contained backpack that lets astronauts move around in space using small nitrogenjet thrusters. In our app, as in the SAFER system, joystick input activates the jetpack thrusters to control either angular or linear acceleration. The application simulates the visual environment outside the international space station.

In addition to developing this cost-effective training system, we also wanted to evaluate how the neutral buoyancy environment underwater impacts sickness and presence during VR use, so we performed an evaluation of the underwater VR experience during jetpack training. Underwater VR systems have been developed previously but evaluation of these systems is lacking. Neutral buoyancy represents an altered sensory environment, and it is not yet known how this environment impacts VR experiences. Research presented here addresses this gap and at the same time presents a VR solution for more cost-effective neutral buoyancy training.

\section{RELATED WORK}

NASA already uses VR and augmented reality (AR) to train astronauts for a variety of tasks that are unique to space missions. Most relevant to the current application, NASA's Virtual Reality Lab has developed a simulator to train astronauts in the use of the SAFER jetpack locomotion system [Space Center Houston 2018]. Users are seated, so the simulation is not multisensory, but only visual. The input joystick device is a replica of the actual SAFER control device, and control replicates dynamics of the SAFER system, as in our application.

To simulate reduced or zero-gravity environments, in addition to the NBL, NASA has developed the Active Response Gravity Offload System (ARGOS). This system senses movements of the user (or the payload) and offloads weight through a system of cables to reduce the amount of weight that must be supported by the user (or payload) itself. NASA's Hybrid Reality Lab has employed the ARGOS system to develop a simulator that trains astronauts to use a system of handrails to move around in simulated zero-gravity [National Aeronautics and Space Administration 2013]. Known as handrail locomotion, this is the most common method by which astronauts move around on the outside of the spacecraft during a spacewalk.

However, to date NASA has not developed an underwater virtual reality system. Previous studies have developed underwater AR for entertainment purposes, such as coral reef snorkeling [Bellarbi et al. 2013; Oppermann et al. 2013; Yamashita et al. 2016], and training purposes, such as commercial diving [Morales et al. 2009]. Underwater AR and mixed reality (MR) have also been explored as educational tools in archaeology [Bruno et al. 2017] and marine science [Chouiten et al. 2012; Vasiljevic, Borovic, and Vukic 2011]. Underwater VR has previously been developed for use in physical therapy and clinical rehabilitation [Costa, Guo, and Quarles 2017; Osone, Yoshida, and Ochiai 2017; Quarles 2015], but these applications involve snorkeling rather than SCUBA diving. Recent research has begun developing means of simulating underwater locomotion in terrestrial environments [Ichikawa et al. 2019] [Jain et al. 2016], with promising results. Underwater VR is also being developed for entertainment purposes in the private sector [BallastVR 2019]. 
Finally, VR in altered/deprived sensory environments is being investigated as a research tool. Researchers in Italy have created and presented a system intended to serve as a means of conducting behavioral neuroscience research [Bellomo et al 2008]. More recently, sensory deprivation has been investigated as a means of facilitating suspension of disbelief, thereby increasing immersion in a given VR game or experience [Mann, Hao, and Werner 2018]. Sensory deprivation therefore may be a useful means of increasing fidelity and presence of any space-based VR experience.

Several of these systems are reviewed and compared in a recent paper that also presents an underwater VR system for SCUBA training [Hatsushika, Nagata, and Hashimoto 2018]. However, presence and sickness are not evaluated. Another previous application describes a system that could potentially accommodate a standardsized SCUBA regulator [Zhang, Tan, and Chen 2016]. However, no information on how users breathe while wearing this system underwater is provided. To our knowledge, no user studies have been reported that evaluate the effectiveness of underwater VR and compare the effects of underwater VR on sickness and presence relative to normal VR.

\section{UNDERWATER HMD DESIGN}

Here we describe development of an underwater VR system for use while SCUBA diving. Our U-HMD is specifically developed for astronaut training purposes where neutral buoyancy is required to approximate the zero-gravity environment of space. We conduct a user study to evaluate the effectiveness of the system for training applications and to examine the effects of underwater VR on sickness and presence.

\subsection{Hardware Design}

We opted for a hardware solution where a smartphone is incorporated into a VR display using attachable lenses. A benefit of this approach is that many smartphones are already waterproof. We used the OTS Guardian full-face SCUBA diving mask as the base of our prototype. This mask was selected in part because the visor is removable. To convert the mask into an HMD (see Fig. 2), we developed a replacement visor in which we mounted a smartphone (Samsung Galaxy S7) and lenses (Homido mini) (see Fig.2 A). The HMD visor was developed by performing a 3D scan of the original visor and importing the scan into a 3D modeling tool called Blender. The 3D model was then modified by extending and flaring the forward portion of the visor to accommodate the smartphone and lenses at the appropriate distance from the eyes of the average user. 3D models of the phone and lenses enabled the development of the visor to achieve exact fits for their real world counterparts. In order to stabilize the phone screen, the visor walls were thickened to increase their rigidity, and a snug cutout in the back wall of the visor was created for the phone. Additional notches were added to accommodate the mounting of the lenses to the phone, to prevent the pressing of the external buttons on the phone, and to allow cables to be plugged into the bottom side of the phone. The visor was then 3D-printed and mounted in the mask. Several iterations were required to fine-tune the design elements described above. Ultimately, the visor was treated using flexible rubber sealant to

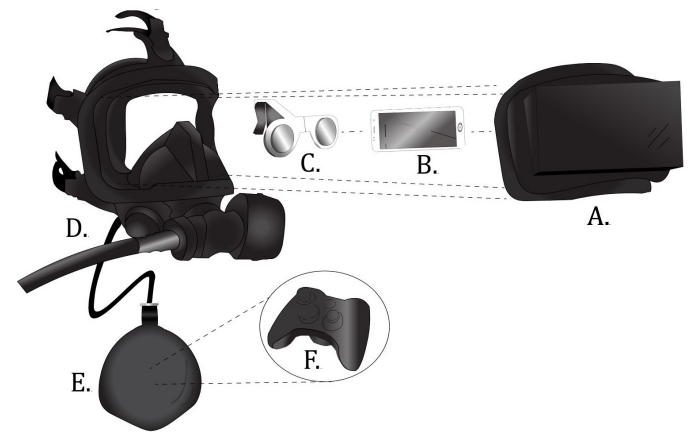

Figure 2: Illustration of underwater HMD components. A modified visor (A) was designed for an OTS Guardian fullface SCUBA mask (D) so that it could accommodate a Galaxy S7 smartphone (B) and Homido clip-on lenses (C). An Xbox 360 Controller $(\mathrm{F})$ was used for input. It was waterproofed by placing it in a latex breathing bag (E).

ensure integrity of fit and waterproofing of the porous 3D-printed material.

For input, we decided to use a wired Xbox 360 controller. NASA's SAFER system relies on a proprietary controller, but this controller costs $\$ 200,000$ and we did not have one available for this project. The Xbox 360 controller is familiar to many people and thus requires little training to be used. We chose a wired solution because wireless signals (e.g. Bluetooth, WiFi) degrade at range underwater [Quarles 2015]. In order to make the controller waterproof, it was placed inside of a latex breathing bag that is conventionally used in the context of anesthesia. Latex was chosen because it easily deforms, and this leads to minimal interference with normal haptic user interface with the controller. The mouth of the bag where the controller's cord exited was sealed closed with a metal washer and silicone. The controller's cord entered the mask through the communication port on the mask, and the entry point was sealed with silicone. A USB-to-MicroUSB adapter was used inside the mask and allowed the controller to be plugged into the phone.

For communication, a $3.5 \mathrm{~mm}$ audio cable was also run to the mask and plugged into the phone. The cord entered through the communication port along with the controller's cord. The other end of the audio cable was plugged into a microphone used by the experimenter above water which allowed for one-way communication from the experimenter to the user. Sound was emitted from the smartphone speaker and was sufficiently audible to the user underwater. Ergonomic fit of the mask to each user was achieved by adding padding where needed when the lenses touched the face or nose, and also by using the changeable nose pads that were provided with the mask. Because the volume of the visor was increased to accommodate the phone and lenses, the air inside the mask led to unwanted buoyancy that pulled the users face toward the surface. To counteract this force, small weights were added to the outside of the visor before each dive.

\subsection{Software Design}

Software was developed to simulate jetpack locomotion. We focused on jetpack locomotion because this is a real task that astronauts are 


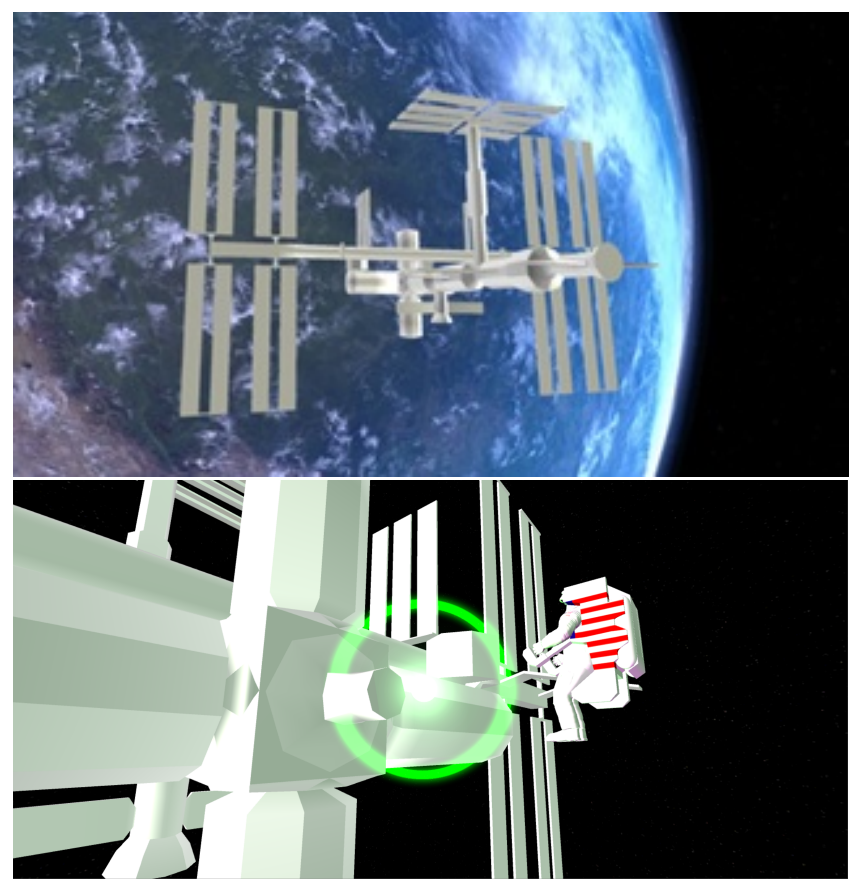

Figure 3: Screenshots of app used to simulate jetpack locomotion. The international space station is shown in orbit around earth as well as the astronaut with jetpack. Participants used jetpack locomotion to navigate through green checkpoint gates.

trained to perform, and yet the requirements for VR simulation are relatively simple in that no haptic interaction is required besides use of the input device. In addition, locomotor tasks are ideal for assessing presence and sickness in VR. The application simulated the visual environment outside the International Space Station (ISS) in orbit around earth (see Figure 3). Within this environment, users are required to use jetpack locomotion to move through a series of checkpoints, shown as green circles. Checkpoints were placed at random locations around the space station approximately equally spaced and they appear one at a time. The application was developed in Unity 2017.1.1 and compiled to an Android APK that was installed on the phone. The models for the simplified International Space Station and astronaut were obtained from the NASA Space Flight assets pack [National Aeronautics and Space Administration 2018]. The mobile VR system had a frame rate of $60 \mathrm{fps}$, and a screen resolution of 1280x1440 per eye. We used lowpolygon models to speed processing. Angular head-motion was tracked by the phone's gyroscope and used to update the view on the screen with a motion-to-photons latency typical of mobile VR systems. Linear head position was not tracked; linear position in the virtual environment could only be controlled via jetpack locomotion.

Jetpack locomotion was controlled via the wired Xbox 360 controller, with two-degree-of-freedom (2DOF) translational movements (fore-aft and left-right) controlled by the left joystick and 2DOF rotational movements (pitch and yaw) controlled by the right

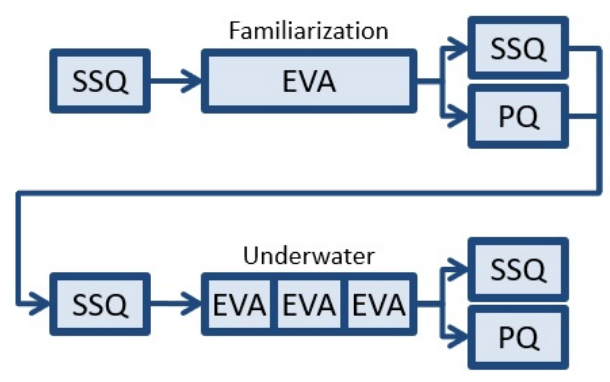

Figure 4: Temporal sequence of data collection. The familiarization session (top) began with administration of the simulator sickness questionnaire (SSQ), followed by 1 block of 5 jetpack locomotion tasks (EVA), followed by repeated administration of the SSQ and the presence questionnaire (PQ). The underwater session (bottom) was conducted on a separate day and followed the same sequence, but with 3 blocks of 5 locomotion tasks.

joystick. For simplicity, we decided not to implement the third degree of freedom for rotation or translation that is available in the SAFER system. Despite this difference, our system is still valid for training dynamic control of jetpack locomotion. The software simulated the dynamics of the SAFER jetpack system with an assumed mass of $337.93 \mathrm{~kg}$ for the astronaut plus spacesuit. When translation input was detected, it delivered $14.24 \mathrm{~N}$ of linear force resulting in acceleration at $0.04 \mathrm{~m} / \mathrm{s}^{2}$. Rotation input delivered $2.938 \mathrm{Nm}$ or $2.26 \mathrm{Nm}$ of torque for pitch and yaw, respectively, resulting in acceleration of $2.819 \mathrm{deg} / \mathrm{s}^{2}$ for pitch and $6.56 \mathrm{degs} / \mathrm{s}^{2}$ for yaw. The software also implemented the SAFER Automatic Attitude Hold functionality which applies thrust to automatically zero angular movement. Matching functionality to zero linear movement was also added to enhance usability. These holding functions were activated by clicking on the appropriate joystick, left for translation, right for rotation.

For communication purposes, a separate Android application was created to run simultaneously with the jetpack simulation software. This application allowed for one-way communication from the experimenter outside the pool via microphone to the participant inside the pool via smartphone speakers. Due to the hardware and software limitations of the Android smartphone, only one-way communication could be implemented. Two-way communication would simultaneously require input from the smartphone's microphone to the external headphones and input from the external microphone to the smartphone's speakers. By default, connecting to the $3.5 \mathrm{~mm}$ jack disables the smartphone's speakers. Through Android API settings, it is possible to bypass the problem and enable input from the external microphone to the smartphone's speakers. However, the Android API is incapable of concurrently enabling communication in both directions.

\section{USER STUDY}

The aim of the user study was to evaluate the functionality of the underwater VR system. In the study, users were required to navigate 
through a series of checkpoints in the simulated environment. We collected data on time-to-completion for each checkpoint as well as data on simulator sickness and presence via questionnaires. We analyzed time-to-completion data to examine whether training was effective, leading in a reduction in time-to-completion by the end of the study. To examine how being underwater influenced user experiences, we compared data on sickness and presence that was reported above water with that reported when using the system underwater.

\subsection{Participants}

Nine participants took part in the study (3F, 6M). Participants were recruited via fliers distributed across campus and in local dive shops. Participants were required to be SCUBA-certified prior to their participation in the study. Participants typically had prior VR experience and non-VR gaming experience. Therefore, they were familiar with the concept of VR and also with the layout of the controller. The study was approved by the university's institutional review board.

\subsection{Procedure}

Each participant first completed a familiarization session above water in the lab. The purpose of this session was to familiarize the participant with the system, to verify that the participant was comfortable wearing the U-HMD, to verify that the participant was capable of performing the required locomotion tasks, and to collect baseline questionnaire data. After being briefed, participants were fitted with the prototype U-HMD and completed one block of jetpack locomotion tasks in which they navigated through five consecutive checkpoints. During this session, participants were seated in a freely-rotating office chair. Before and after completion of the task, the Simulator Sickness Questionnaire (SSQ) [Kennedy et al. 1993] was given to assess presence of symptoms such as nausea and discomfort. Total scores and subscores were computed as described in [Kennedy et al. 1993]. We also assessed user experience via the Presence Questionnaire (PQ) [Witmer and Singer 1998] after they completed the block (see Figure 4).

To control for any lingering effects of VR sickness, participants completed the underwater portion of the user study at least 24 hours later (i.e, the next day). The structure of the underwater session resembled that of the training session (Figure 4). It began with completion of the SSQ. This was followed by completion of the jetpack locomotion task while floating underwater with neutral buoyancy. However, instead of completing only one block of 5 checkpoints, participants completed three consecutive blocks of 5 checkpoints each while underwater. This allowed us to measure any improvement in performance across the blocks. Following the dive, participants completed the SSQ as well as the PQ.

The logistics surrounding the underwater session were much more involved than for the familiarization session and required both an experimenter above water and a companion diver. Experiments took place at the university's competitive diving tank and began with inspection and setup of dive equipment for both the participant as well as the companion diver. The diving tank had a depth of approximately 4.57 meters ( 15 feet). The primary task of the companion diver was to ensure the safety of the participant.

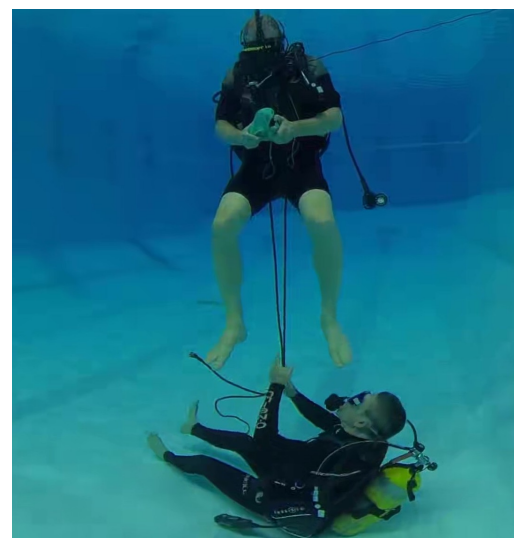

Figure 5: Photo of participant performing the experiment underwater with the companion diver also visible.

During the experiment, participants were unable to see outside the U-HMD so the companion diver monitored their position, orientation, and air supply. Both divers were outfitted with a vest-type buoyancy control device (BCD) and a 7 litre aluminum cylinder with a standard starting pressure of 3000 PSI. These were connected to the DIN-standard adapter and regulator using an A-clamp pillar valve. An electronic dive computer was used to keep track of air, depth, and total dive time for both divers. All dive equipment was borrowed free of charge from a local dive shop.

Before entering the water, the companion diver and participant reviewed basic hand signals for diving that would allow for communication during the experiment [Recreational Scuba Training Council 2014]. Although the participant could not see any signals by researchers, the participant could provide signals to researchers to indicate a change in status. A fist with the thumb extended upward indicated that immediate ascension to the surface was required. This was also used to indicate completion of the experiment. Participants were instructed to create a circle with their thumb and forefinger, with the remaining three fingers extended to indicate that they felt comfortable and could proceed without issue. With a flat hand rotating back and forth, participants could indicate that something was wrong. Participants would not be immediately returned to the surface with this signal unless also accompanied by the extended thumb and fist signal. After seeing the rocking hand, the companion diver was required to periodically reassess the state of the participant by tapping on their arm or leg. This prompted the participant to give another hand signal to indicate their status.

After putting on and testing all dive equipment, the participant was seated on the edge of the pool, and the U-HMD was secured. The companion diver then guided the participant into the water and adjusted the buoyancy of themselves and the participant via inflation and deflation of both BCDs. In order to ensure neutral buoyancy, participants maintained minimally positive buoyancy while tethered to the researcher, who was negatively buoyant (see Figure 5). The tether was looped through the front D-rings on the $\mathrm{BCD}$ of the participant and managed by the researcher. Once comfortably settled in this position, the participant began the experiment. The companion diver continued to monitor buoyancy 


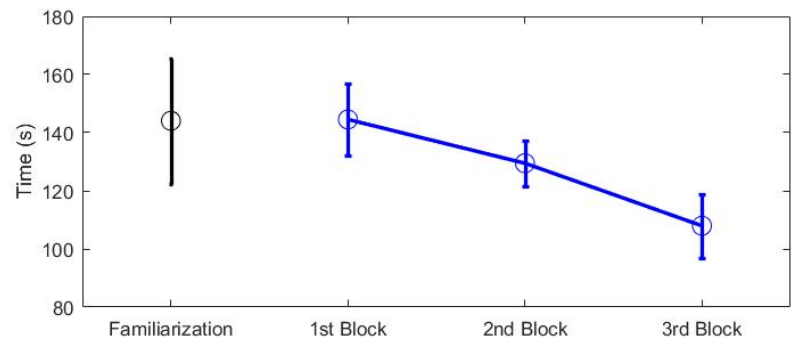

Figure 6: Average duration required to pass each gate using jetpack locomotion during the familiarization block (black) and the three underwater blocks (blue). Each block consisted of five gates. Each point is the across-subject mean of each subject's average gate completion time for that block. Error bars show standard error of the mean.

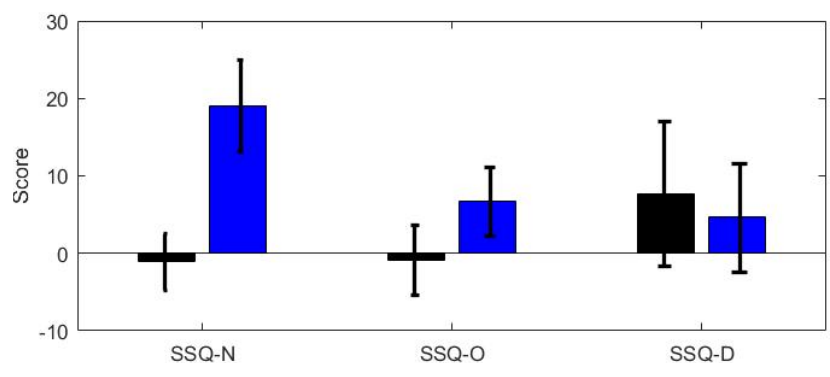

Figure 7: Change in VR sickness $(\triangle \mathrm{SSQ})$ reported in familiarization (black) and underwater (blue) sessions for nausea $(\mathrm{N})$, oculomotor $(\mathrm{O})$, and disorientation (D) symptoms. Error bars show standard error of the mean.

and check air every 3 to 5 minutes until the session was over. The total dive time was 20 to 40 minutes, depending on the participant.

\subsection{Results}

The underwater VR system was successfully used to perform spacewalk training. Across blocks, there was a reduction in the time it took participants to navigate through the gates using jetpack locomotion (see Figure 6). During training and during the first underwater block, participants required more than 140 seconds, on average, to complete each gate. By the end of the third block, average completion time was less than 110 seconds. The reduction in completion time across the underwater blocks was significant $\left(\boldsymbol{F}_{2,8}=8.45, p<.05\right)$.

In addition to assessing training, data was collected on simulator sickness before and after the familiarization session and before and after the underwater session. All statistical analyses were performed on the change in total SSQ scores ( $\triangle \mathrm{SSQ}$ ), defined as the total SSQ score for each subject after each session minus the total SSQ score for that subject before each session (post minus pre). Overall, $\triangle \mathrm{SSQ}$ values in the underwater session $(M=113.91, S D=159.67)$ were not significantly different than $\triangle \mathrm{SSQ}$ values in the familiarization session $(\mathrm{M}=21.81, \mathrm{SD}=149.14 ;(\boldsymbol{t}(8)=-1.009, p>.05)$.The underwater session did not lead to significantly more sickness than the familiarization session.

To get more insight on possible sickness induced by our system, we also evaluated the SSQ subscales (see Figure 7) for nausea (N), oculomotor function (O), and disorientation (D). Reports of symptoms associated with nausea were significantly greater following the underwater session compared to the familiarization session, as indicated by the greater $\triangle \mathrm{SSQ}-\mathrm{N}$ value $(\boldsymbol{t}(8)=-2.562, p<.05)$ Reports of oculomotor discomfort $(\triangle \mathrm{SSQ}-\mathrm{O})$ did not differ significantly between sessions $(\mathrm{t}(8)=-1.061, \mathrm{p}=0.320)$, nor did reports of disorientation $(\triangle \mathrm{SSQ}-\mathrm{D})(\boldsymbol{t}(8)=0.220, p>.05)$.

Table 1: Presence questionnaire results including scores for subscales.

\begin{tabular}{|l|r|r|r|r|}
\hline \multicolumn{1}{|c||}{} & \multicolumn{2}{|c|}{ Familiarization } & \multicolumn{2}{c|}{ Underwater } \\
\hline PQ subscales & M & SD & M & SD \\
\hline Realism & 35.63 & 5.4 & 36.38 & 4.5 \\
\hline Possibility to Act & 24.63 & 2.3 & 23.63 & 2.8 \\
\hline Quality of interface & 15.63 & 2.6 & 16.63 & 3.2 \\
\hline Possibility to examine & 17.38 & 2.4 & 18.25 & 2.3 \\
\hline Self-evaluation & 11.38 & 1.4 & 12.00 & 1.7 \\
\hline Total scores & 104.63 & 9.5 & 106.88 & 12.5 \\
\hline
\end{tabular}

We were also interested in the effect of neutral buoyancy on the feeling of presence in the virtual environment. PQ scores trended towards slightly higher in the underwater session $(M=106.88, S D$ $=12.529)$ compared to the familiarization session $(\mathrm{M}=104.63, \mathrm{SD}=$ 9.471), but this difference was not significant $(\boldsymbol{t}(7)=-1.107, p>$ .05)

Analysis of the PQ subscales shows no significant differences in realism $(\boldsymbol{t}(7)=-1.655, p>.05)$, possibility to act $(\boldsymbol{t}(7)=1.871, p>$ $.05)$, quality of interface $(\boldsymbol{t}(7)=-.581, p>.05)$, possibility to examine $(\boldsymbol{t}(7)=-1.178, p>.05)$, or self-evaluation of performance $(\boldsymbol{t}(7)=-1.106, p>.05)$. Means and standard deviations of these measures can be found in Table 1. One participant was excluded from $P Q$ analyses because they did not complete all pages of the questionnaire. Questions on the PQ regarding haptics and sound, along with one item "How much did your experiences in the virtual environment seem consistent with your real world experiences?", were excluded as they were either not included in the VR system (haptics and environmental sounds), or impossible for participants to answer (most people have not visited space).

\section{DISCUSSION}

We demonstrate how a full-face dive mask can be modified to build a VR system for use while SCUBA diving. We used this system to develop a training simulation for jetpack locomotion, because astronauts train for spacewalks underwater. A user study showed that the system was effective in training participants, leading to a reduction in time to complete jetpack locomotion tasks. SSQ data showed greater reports of nausea after use of the VR system underwater compared to above water, but no other differences in reported sickness symptoms. Use of VR underwater did not have a significant impact on presence as evaluated with the PQ. 


\subsection{Evaluation of VR Sickness and Presence}

Spatial orientation is reconstructed based on multiple sources of sensory input [Howard 1982], so it is reasonable to expect alterations in perception of spatial orientation, sickness, and presence during VR use underwater because somatosensory and proprioceptive cues to orientation and movement are rendered uninformative. A primary focus of the current study was therefore to perform an evaluation of VR experiences in this altered sensory environment. In general, our application did not elicit high levels of VR sickness, but greater levels of VR sickness were observed following the underwater session, particularly on the nausea subscale of the SSQ, which assesses symptoms such as stomach awareness, increased salivation, sweating, and general discomfort. However, we cannot conclude that VR use was driving these elevated scores. Increased sickness could be the result of exposure to neutral buoyancy alone. Alternatively, it could be that exposure to the SCUBA experience, including prolonged breathing of compressed air in an increased pressure environment, elicits higher levels of VR sickness. Finally, VR exposure time while underwater was three times longer (i.e. 3 blocks of 5 underwater compared to 1 block of 5 above), so duration of exposure could be driving this effect. On balance, it is encouraging that higher levels of VR sickness were not observed while using VR in a neutral buoyancy environment.

Regarding presence, we expected increased levels of presence following exposure to VR underwater because somatosensory and proprioceptive cues are rendered uninformative during neutral buoyancy and we expected visual cues to become more dominant in this context. However, there was no significant difference in results of the PQ between familiarization and underwater sessions. The result is despite qualitative feedback from participants who were enthusiastic about the immersion they experienced during the underwater session. A reason why we may not have detected statistical significance could be due to the small number of participants that we included in our study. Not only did we require participants to be SCUBA certified, running a single participant through the underwater experiment was resource-intensive and limited the number of participants that could be included in our study.

Another possible explanation is that the PQ is not well-suited for assessing experiences like spacewalks that are wholly foreign and novel to users. As we removed some items from the PQ, the validity of the PQ may have been compromised; which may partially explain the lack of concurrent validity between the PQ's assessment and the positive reports made by some participants. However, many of the PQ's questions focus on how the simulated experience compares to the user's real-life experience: a comparison which was difficult for all of our participants to make. An avenue for future work may be development of a questionnaire that is more appropriate for assessing presence in novel environments. Future analysis of user experience may also be improved by having a structured, qualitative debrief that could assess presence, including questions about perceived weightlessness, separately from the PQ or any other questionnaire.

Some participants also reported factors that may have detracted from presence while underwater. Specifically, there was some vibration of the mask when exhaling when using the U-HMD with a regulator, and this led to a perception of the world periodically shaking or vibrating. In the future, it would be helpful to find a way to eliminate this vibration. One solution would be to use a conventional dive mask, rather than a full-face mask, so that the regulator is detached from the mask; similar to prototypes described in previous literature [Hatsushika, Nagata, and Hashimoto 2018; Osone, Yoshida, and Ochiai 2017; Zhang, Tan, and Chen 2016]. This decision must be weighed against the advantages of the full-face mask, specifically the ability to talk while underwater. Another factor that may have detracted from presence while underwater is the bubbles of the companion diver. Because the companion diver was often directly below the participant, the bubbles were sometimes distracting. To avoid this in future, a different method could be devised for maintaining neutral buoyancy.

\subsection{U-HMD suitability for EVA training}

The reduction in time to completion for the jetpack locomotion task shows that training was successful, and participants learned to use the joystick interface to control their movement in the virtual environment. While this is reassuring, it is likely that we would have observed this result after repeated exposure above water. Nevertheless, conducting the study underwater allowed us to demonstrate the feasibility of the underwater VR system and to assess the impact of underwater VR use on sickness and presence.

There are several developments that could enhance this technology going forward. One feature that was missing was real physical movement consistent with the visually simulated movement. This leads to a conflict between visually simulated motion and motion signaled by the vestibular organ in the inner ear. This sensory conflict could be driving feelings of nausea reported by participants after completing the underwater phase of our experiment. However, peak linear acceleration experienced by users in the current study falls below average human detection thresholds shown in previous work [MacNeilage et al. 2010]. Conversely, peak angular acceleration experienced by participants exceeded previously recorded thresholds for transient rotations [MacNeilage, Turner, and Angelaki 2010]. It is possible that the mismatch between visual and vestibular senses here is driving some feelings of nausea, but unlikely given mechanics of the semicircular canals. The semicircular canals tend to be most sensitive to transient rotations; it is unclear if sustained rotation would elicit similar detection thresholds (and thus, subsequent sensory mismatch).

Nevertheless, in order to overcome a limitation like this, it would be necessary to develop a method to physically move users that is consistent with the jetpack locomotion commands and the visual scene. One such method is using water currents, either applied externally (jets) or applied via an aquatic jetpack worn by the user. Promising research has shown an ability to take flow measurements using transparent tracer particles [Yamashita et al. 2018], which would have great utility for tracking any sort of underwater movement experienced. Another possibility is using humans to provide feedback, as demonstrated in previous research [Cheng et al. 2014].

More generally, if underwater VR is going to replace the NBL, several additional developments are needed. Most importantly, our users did not gain experience maneuvering in spacesuits. A solution would be to build a virtual or augmented reality display into the 
visor of the NBL spacesuits. Another limitation of our system was that users were not able to see their own bodies in the simulation; they only saw a static representation of the spacesuit that did not move in agreement with their own body movements. While this is a limitation of many VR systems, overcoming this limitation, for example by tracking and rendering the hands and other body parts, would likely improve the simulation tremendously.

Finally, a strong advantage of the NBL over our underwater VR system is the ability to interact haptically with the environment. Our simulation prioritized low cost over fidelity, and a lack of realistic haptic interaction and feedback was a consequence of this design philosophy. If a VR system is to replace the NBL, it will be necessary to find a way to provide haptic feedback underwater in conjunction with virtual environment interaction that is consistent with interaction demands of the EVA training environment, including, for example, handrail locomotion and tool use. This haptic fidelity could be improved by introducing props for the participant to use (such as handrails) when completing a virtual task.

\subsection{Design Considerations}

Due to the potential of underwater VR for a variety of applications, from training to entertainment, it is useful to consider general design challenges in developing VR for underwater usage. We chose to mount our display inside the mask, similar to previous applications [Osone, Yoshida, and Ochiai 2017; Zhang, Tan, and Chen 2016]. Other applications [Quarles 2015] have used a waterproof display and mounted it outside the mask. This decision depends on the application. When diving to any significant depth, an in-mask solution is preferable because commercially available waterproof displays are not designed to work at significant depths.

The decision to mount the display inside the mask required designing the visor to achieve a snug fit. It was necessary to try several iterations of the visor before a good solution was achieved with the display mounted in the right position. It was also necessary to devise a method to hold the phone in place; ultimately, we used spring clips fabricated from wire. With our implementation, it was not possible to adjust the position of the lenses, display, or the interpupillary distance, which led to suboptimal fits for some users. The ability to customize the display to the user would be a desirable feature in future implementations.

For input we settled on a wired controller, but this was not an optimal solution. The wire was cumbersome, and we had problems with connecting it to the phone due to the confined space in the mask and the need for an adapter. In addition, the wire frayed after extended use. For these reasons, a wireless input solution would be preferable. Unfortunately, conventional wireless signals such as Bluetooth and Wi-Fi cannot travel long distances in water [Quarles 2015]. A general purpose method for wireless underwater communication would be helpful for the purposes of input, vocal communication, and other uses.

Maintaining low cost was the chief constraint of hardware and software design. In all, the total cost of all materials needed for assembly of the U-HMD amounted to less than $\$ 2,000$ dollars, with the dive mask being the most expensive component. While this low-cost strategy has benefits, it sacrifices the high fidelity seen in other, more expensive simulator systems. Some improvement could be achieved, for example by incorporating a higher quality HMD. Other improvements, such as realistic haptic feedback and physical motion stimulation, will be more challenging to implement. The main benefit of our system is that hardware and software are easy to disseminate and recreate outside of our lab. The 3D model of the 3D-printed visor has been uploaded to thingiverse.com to allow the research community to build their own U-HMDs and modify it to fit different lenses and smartphones. Regardless, any future design iteration would not be complete without close consideration of this fidelity-cost tradeoff.

\section{CONCLUSION}

To our knowledge, we present the first data evaluating the effect of underwater VR on VR sickness and presence. In addition, the use of underwater VR for astronaut training is novel and has potential to be a training paradigm used to augment or replace current methods at a fraction of the cost. We document several challenges unique to standalone SCUBA-based implementations of VR, along with other challenges that generalize to all types of aquatic VR and AR systems. These observations should aid in development and evaluation efforts going forward, as we anticipate a wide range of potential future applications for underwater VR and AR.

\section{ACKNOWLEDGMENTS}

The authors would like to thank the Sierra Diving Center and their staff for donating time, equipment, and expertise to this project. The authors also wish to thank the anonymous reviewers for their comments and suggestions, as well as the pool staff at the Lombardi Recreation Center. This material is based upon work supported by the National Aeronautics and Space Administration under Grant No.: NNX15AIO2H.

\section{REFERENCES}

BallastVR. 2017. About ballast. Available: https://ballastvr.com/about/. [Accessed Sept. 20 2019].

Abdelkader Bellarbi, Christophe Domingues, Samir Otmane, and Samir Benbelkacem. 2013. Augmented reality for underwater activities with the use of the dolphyn.. In International Conference on Networking, Sensing and Control (ICNSC), 2013 10th IEEE International Conference on. IEEE, 409-412.

Eric. Berger. 2017. Why is NASA renting out its huge astronaut pool? To keep the lights on. Available: https://arstechnica.com/science/2017/02/as-it-seeks-to-parecosts-nasa-opens-its-historic-facilities-to-private-companies/. [Accessed Sept. 20 2019]

Fabio Bruno, Antonio Lagudi, Gerardo Ritacco, Panagiotis Agrafiotis, Dimitrios Skarlatos, Jan Cejka, Pavel Kouril, Fotis Liarokapis, Oliver Philpin-Briscoe, Charalambos Poullis, and Sudhir. Mudur. 2017. Development and integration of digital technologies addressed to raise awareness and access to European underwater cultural heritage. An overview of the H2020 i-MARECULTURE project.. In OCEANS 2017 Aberdeen. IEEE.

Mehdi Chouiten, Christophe Domingues, Jean-Yves Didier, Samir Otmane, and Malik Mallem. 2012. Distributed mixed reality for remote underwater telerobotics exploration.. In 2012 Virtual Reality International Conference (VRIC). VRIC, 409-412.

Raphael Costa, Rongkai Guo, and John Quarles. 2017. Towards usable underwater virtual reality systems. IEEE 2017 Virtual Reality 3 (2017), 271-272.

Recreational Scuba Training Council. 2014. Common hand signals for recreational scuba diving. Online $p d f$ Available http://www.neadc.org/ CommonHandSignalsforScubaDiving.pdf. [Accessed Sept. 20 2019].

Space Center Houston. 2018. How NASA uses virtual reality to train astronauts. Available: https://spacecenter.org/how-nasa-uses-virtual-reality-to-train-astronauts/. [Accessed Sept. 20 2019].

Robert S Kennedy, Norman E Lane, Kevin S Berbaum, and Michael G Lilienthal. 1993. Simulator sickness questionnaire: An enhanced method for quantifying simulator sickness. The international journal of aviation psychology 3, 3 (1993), 203-220. 
Moe Lauchert and John David. Narramore. 2017. SCUBA diving in the NASA neutral buoyancy lab. Available: https://scubadiverlife.com/scuba-diving-nasa-neutralbuoyancy-laboratory/. [Accessed Sept. 20 2019].

R. Morales, P. Keitler, P. Maier, and G. Klinker. 2009. An underwater augmented reality system for commercial diving operations.. In MTS/IEEE Oceans. IEEE, 409-412.

NASA. 2013. Active response gravity offloading system. Available:

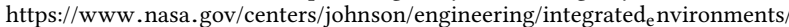
active $_{\mathrm{r}}$ esponse $_{\mathrm{g}}$ ravity/. [Accessed Sept. 20 2019]

NASA. 2018a. Facts about spacesuits and spacewalking. Available: https:// www.nasa.gov/audience/foreducators/spacesuits/facts/index.html. [Accessed Sept. 20 2019]

NASA. 2018b. What is a spacewalk? Available: https://www.nasa.gov/audience/ forstudents/k-4/stories/nasa-knows/what-is-a-spacewalk-k4.html. [Accessed Sept 20 2019].

NASA. 2018c. Unity Store: NASA space flight assets. Available: https: //assetstore. unity.com/packages/3d/vehicles/space/nasa-space-flight-assets- 756 [Accessed Sept. 20 2019].

Ian P. Howard. 1982. Human visual orientation. Chichester, Sussex, England and New York, John Wiley and Sons.

Majed Al Zayer, Paul MacNeilage, and Eelke Folmer. 2018. Virtual locomotion: a survey. In IEEE transactions on visualization and computer graphics.

Denik Hatsushika, Kazuma Nagata, and Yuki Hashimoto. 2018. Underwater VR Experience System for Scuba Training Using Underwater Wired HMD. In OCEANS 2018 MTS/IEEE Charleston.

Leif Oppermann, Lisa Blum, Jun-Yeong Lee, and Jung-Hyub Seo. 2013. AREEF Multiplayer underwater augmented reality experience. In International Games Innovation Conference, 2013 IEEE International. IEEE, 199-202.

Hiroyuki Osone, Takatoshi Yoshida, and Yoichi Ochiai. 2017. Optimized HMD System for Underwater VR Experience. In ACM SIGGRAPH 2017 Posters (SIGGRAPH '17). ACM, New York, NY, USA, Article 25, 2 pages. DOI : http://dx.doi.org/10.1145/ 3102163.3102232

J. Quarles. 2015. Shark punch: A virtual reality game for aquatic rehabilitation. In 2015 IEEE Virtual Reality (VR). 265-266. DOI : http://dx.doi.org/10.1109/ VR.2015.7223397

Antonio Vasiljevic, Bruno Borovic, and Zoran Vukic. 2011. Augmented Reality in marine applications. Brodogradnja 2, 62 (2011), 136-142.

Bob G Witmer and Michael J Singer. 1998. Measuring presence in virtual environments:
A presence questionnaire. Presence: Teleoperators and virtual environments 7,3 (1998), 225-240.

Shotaro Ichikawa, Isamu Endo, Yuki Onishi, Aoi Suzuki, Daigo Hayaski, Anri Niwano, Akiyuki Ebi and Yoshifumi Kitamura. 2019. Be bait!: A unique fishing experience with hammock-based underwater locomotion method. In 2019 IEEE Virtual Reality (VR). 265-266

Dhruv Jain, Misha Sra, Jingru Guo, Rodrigo Marques, Raymond Wu, Justin Chiu, Chris Schmandt. 2016. Immersive scuba diving simulator using virtual reality. In 2016 User Interface Software and Technology Proceedings. 729-739

Shogo Yamashita, Xinlei Zhang, Takashi Miyaki, and Jun Rekimoto. 2016. Aquacave: an underwater immersive projection system for enhancing the swimming experience. ICAT-EGVE (2016)

Wenlong Zhang, Chek Tien Tan, and Tim Chen. 2016. A Safe Low-cost HMD for Underwater VR Experiences. In SIGGRAPH ASIA 2016 Mobile Graphics and Interactive Applications (SA '16). ACM, New York, NY, USA, Article 12, 2 pages. DOI : http://dx.doi.org/10.1145/2999508.2999519

Steve Mann, Max Lv Hao, and Jeremy Werner. 2018. Virtual reality games in sensory deprivation tanks. In 2018 IEEE Games, Entertainment, Media Conference (GEM). 63-70.

A. Bellomo, M. Benassai, F. Lacquaniti, G. Mascetti, V. Maffei, W. L. Miller, and M. Zago. 2008. Virtual reality in neutral buoyancy (VRNB). Poster session presented at: 3rd National Congress of ISSBB - Italian Society for Space Biomedicine and Biotechnology. 2-3 April 2008, Udine University, Italy.

Shogo Yamashita, Shunichi Suwa, Takashi Miyaki, and Jun Rekimoto. 2018. Water flow measurement technology assessing spatial user interaction in an underwater immersive virtual reality environment. In 2018 IEEE Virtual Reality and 3D User Interfaces (VR). 785-786.

Paul MacNeilage, Martin Banks, Gregory DeAngelis, and Dora Angelaki. 2010. Vestibular heading discrimination and sensitivity to linear acceleration in head and world coordinates. In Journal of Neuroscience, 30 (27). 9084-9094.

Paul MacNeilage, A. H. Turner, and Dora Angelaki. 2010. Canal-otolith interactions and detection thresholds of linear and angular components during curved-path self-motion. In fournal of Neurophysiology, 104 (2). 765-773.

Lung-Pan Cheng, Patrick Luehne, Pedro Lopes, Christoph Sterz, and Patrick Baudisch. 2014. Haptic turk: a motion platform based on people. In Proceedings of the SIGCHI Conference on Human Factors in Computing Systems (CHI '14). ACM, New York, NY, USA, 3463-3472. 